\title{
Electromagnetically induced transparency in rubidium vapor prepared by a comb of short optical pulses
}

\author{
Vladimir A. Sautenkov, ${ }^{1,3}$ Yuri V. Rostovtsev, ${ }^{1}$ C. Y. Ye, ${ }^{1}$ George R. Welch, ${ }^{1}$ Olga Kocharovskaya, ${ }^{1,4}$ and Marlan O. Scully ${ }^{1,2}$ \\ ${ }^{1}$ Department of Physics and Institute for Quantum Studies, Texas A\&M University, College Station, Texas 77843, USA \\ ${ }^{2}$ Department of Chemistry, Princeton University, Princeton, New Jersey 08544, USA \\ ${ }^{3}$ Lebedev Physical Institute, RAS, Moscow 119991, Russia \\ ${ }^{4}$ Institute of Applied Physics, RAS, Nizhny Novgorod 603120, Russia
}

(Received 29 June 2004; published 14 June 2005)

\begin{abstract}
It was shown by Kocharovskaya and Khanin [Sov. Phys. JETP 63, 945 (1986)] that a comb of optical pulses can induce a ground-state atomic coherence and change the optical response of an atomic medium. In our experiment, we studied the propagation of a comb of optical pulses produced by a mode-locked diode laser in rubidium atomic vapor. Electromagnetically induced transparency (EIT) was observed when the pulse repetition rate is a subharmonic of the hyperfine splitting of the ground state. The width of the EIT resonance is determined by the relaxation rate of the ground-state coherence. Possible applications to magnetometery, atomic clocks, and frequency chains are discussed.
\end{abstract}

DOI: $10.1103 /$ PhysRevA.71.063804

PACS number(s): 42.50.Gy, 42.60.Fc, 42.62.Eh

\section{INTRODUCTION}

Coherent effects such as electromagnetically induced transparency (EIT) and coherent population trapping (CPT) [1-3] attract a lot of attention because of their ability to suppress linear absorption and enhance the nonlinear response of a resonant medium. These complementary coherent effects impact techniques such as high-precision spectroscopy [4-7], atomic clocks [8,9], nonlinear interaction with weak light fields at the single-photon level [11-13], greatly reduced phase-matching requirements [14], large Kerr nonlinearities [15], etc.

CPT was first reported in Ref. [16] where the elimination of resonant fluorescence of sodium atoms was observed under conditions where the mode spacing of a multimode dye laser was equal to the ground-state splitting. A description of other experiments with cw lasers can be found in the review [2]. CPT has also been observed in the pulsed regime [17].

Growing interest in EIT was stimulated by Harris's theoretical work [18]. EIT has been successfully demonstrated in different experiments: in continuous-wave and pulsed regimes [18], with atomic and molecular gases (at room temperature $[19,20]$ or with cold atoms [21]), with solids doped by rare-earth ions [22] and semiconductor quantum wells [23], and for different wavelengths ranging from $\gamma$ rays to microwaves $[18,19,24,25]$.

Recently, frequency chains based on femtosecond lasers were developed [26]. One approach in realizing a frequency chain is stabilization of the pulse repetition rate by microwave $\mathrm{Cs}$ or $\mathrm{Rb}$ standards [26]. By using the approach developed by Kocharovskaya and Khanin [1], it is possible to create an all-optical reference frequency chain [7].

As was predicted in Ref. [1], a comb of short optical pulses interacting with a three-level atom can effectively excite coherence between ground states $b$ and $c$ (see Fig. 1) when the ground-state splitting is a multiple of the pulse repetition frequency $1 / T$, namely, $w_{\mathrm{cb}}=2 \pi p / T$, where $p$ is an integer number. If the intensity of the laser radiation is suf- ficiently high, coherence is excited, and the medium becomes transparent to the laser field.

In this paper, we experimentally show the possibility of EIT in a resonant atomic medium by using a mode-locked diode laser generating a comb of picosecond pulses.

\section{EXPERIMENTAL SETUP AND OBTAINED RESULTS}

The experimental setup is shown schematically in Fig. 2. We use an actively mode-locked external-cavity diode laser tuned to the vicinity of the $5 S_{1 / 2} \rightarrow 5 P_{3 / 2}\left(D_{2}\right)$ transition of ${ }^{85} \mathrm{Rb}$ to produce a comb of short optical pulses. Properties of mode-locked diode lasers are described in the review [27]. The laser assembly is similar to the Littman-Metcalf configuration [28]. It is a three-mirror laser cavity that consists of an antireflection-coated diode laser, a collimation lens, a diffraction grating $\left(1200\right.$ grooves $/ \mathrm{mm}$, blaze angle $\left.37^{\circ}\right)$, and external mirror. The spectral width of optical feedback is chosen as broad as $20 \mathrm{GHz}$ which is more than the hf splitting of ${ }^{85} \mathrm{Rb}$. Mode separation is tuned by variation of the cavity length to $506 \mathrm{MHz}$ which is close to one-sixth of the hf splitting (sixth subharmonic). Mode-locked operation is achieved by modulating the diode laser current near this fre-

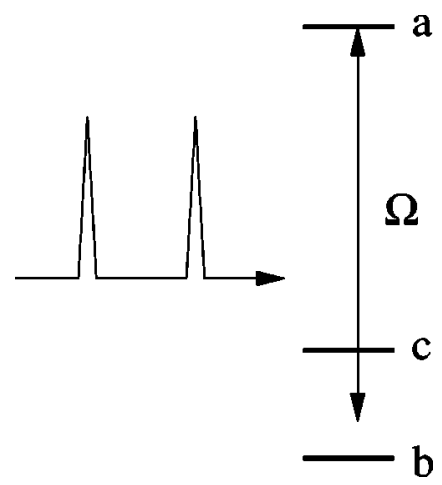

FIG. 1. Schematic of atomic levels. 


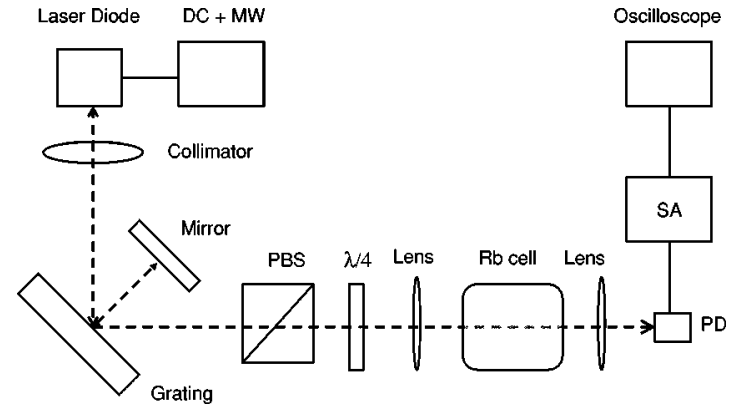

FIG. 2. Schematic of the experimental setup. PBS, polarizing beam splitter.

quency. When the modulation frequency is changed, the repetition rate is also changed. The spectral distribution of our laser covers the hyperfine components of the ${ }^{85} \mathrm{Rb}$ ground state $5 S_{1 / 2}$ (wavelength $780 \mathrm{~nm}$, ground-state hyperfine splitting $3035.7 \mathrm{MHz}$ ).

The laser pulses are measured by an optical sampling oscilloscope. Typical pulses are shown in Fig. 3. The time resolution of the oscilloscope is about 200 ps. One can see that the duration of the observed pulses is defined by the resolution of the oscilloscope. It is clear that the duration of observed pulses is less than 200 ps.

The laser beam is sent into a glass cell $(l=2.5 \mathrm{~cm})$ with the natural abundance of ${ }^{87} \mathrm{Rb}$ and ${ }^{85} \mathrm{Rb}$. To increase the density of rubidium atoms, the cell is heated to $60{ }^{\circ} \mathrm{C}$. The cell is installed in a three-layer magnetic shield. The transmission of the cell was studied by using a heterodyne technique. A beat note at frequencies close to the ground-state hf splitting was recorded. The signal from the fast photodiode (PD) is sent to a microwave spectroanalyzer (SA) which is used as a narrow-band microwave amplifier with central frequency 3035.7 MHz and bandwidth $3 \mathrm{MHz}$. The signal from the spectroanalyzer is recorded by a digital oscilloscope. When the modulation frequency is scanned around $506 \mathrm{MHz}$, the variation of transmitted laser power is observed.

The observed EIT resonance for $0.2 \mathrm{~mW}$ average power is presented in Fig. 4(a). To reduce the possible field broadening, we decreased the average power to $0.05 \mathrm{~mW}$. The result is shown in Fig. 4(b). The amplitudes of resonances are normalized in order to demonstrate the small difference between

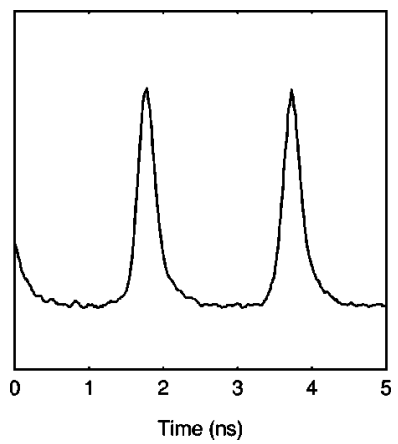

FIG. 3. Optical pulses generated by the mode-locked diode laser. Period between pulses $T$ is about $2 \mathrm{~ns}$ which is six times larger than the period of oscillation of the coherence between sublevels of the ground state.

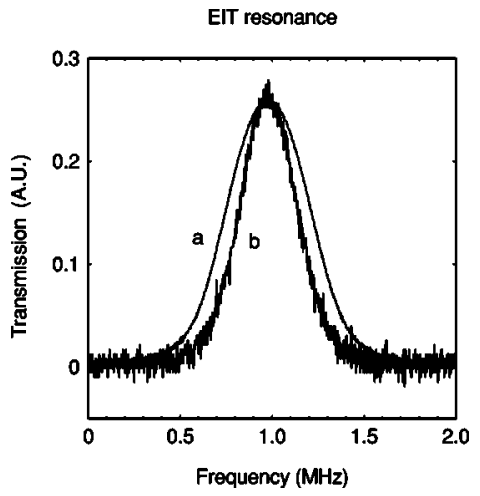

FIG. 4. Transmission of laser radiation vs frequency of repetition rate $(1 / T)$ for short pulses. The repetition rate changes around $506 \mathrm{MHz}$, which is one-sixth of the hf splitting (sixth subharmonic).

the spectral widths [full width at half maximum FWHM 0.5 and $0.37 \mathrm{MHz}$ measured by the ratio of amplitudes]. One can see that we worked with low laser power and spectral broadening of the EIT resonances can be attributed mainly to the interaction time (time of flight of atoms through the laser beam). With a buffer gas cell, one can dramatically reduce the spectral width of the EIT resonance due to a longer interaction time. For instance, Brandt et al. observed CPT resonance with a $40 \mathrm{~Hz}$ width [5].

The amplitude of pulses propagating through the cell depends on the repetition rate $1 / T$. When the repetition rate corresponds to a subharmonic of the hf splitting, $w_{\mathrm{cb}}$ $=12 \pi / T$, the absorption of the cell decreases, i.e., EIT for the comb of optical pulses is observed.

This experimental result can be easily understood by invoking a so-called dark state, a linear combination between sublevels of ground states which is decoupled from the external field. Indeed, the Hamiltonian of the system in the interaction picture is given by

$$
\hat{V}=\Omega_{1}(t)|b\rangle\left\langle a\left|+e^{i p \omega_{\mathrm{cb}} t} \Omega_{2}(t)\right| c\right\rangle\langle a|+\text { H.c. },
$$

where $\Omega_{1}(t)=\wp_{a b} E(t) / \hbar$ and $\Omega_{2}(t)=\wp_{a c} E(t) / \hbar$ are the Rabi frequencies; $\wp_{a b}$ and $\wp_{a c}$ are the dipole moments of corresponding transitions; $\Delta$ is the hf splitting; $E(t)$ is the laser field. The field is periodic, so a Fourier transformation can be made. The Hamiltonian can be rewritten as

$$
\hat{V}=\sum_{p} e^{i p \Omega t}\left(\Omega_{1, p}|b\rangle\left\langle a\left|+\Omega_{2, p} e^{i p \omega_{c b} t}\right| c\right\rangle\langle a|+\text { H.c. },\right.
$$

where $\Omega$ is the frequency corresponding to the time between pulses $\Omega=2 \pi / T ; \Omega_{1, p}$ and $\Omega_{2, q}$ are the Rabi frequencies corresponding to the $p$ th and $q$ th Fourier components of the field.

Let us assume that the repetition rate of the pulses is a multiple of the hf splitting

$$
\omega_{\mathrm{cb}}=Q \Omega
$$

then the Hamiltonian can be rewritten in the form 


$$
\hat{V}=\sum_{p} e^{i p \Omega t} \sqrt{\Omega_{1, p}^{2}+\Omega_{2, p-Q}^{2}}\left|B_{p}\right\rangle\langle a|+\text { H.c. },
$$

where we introduce the states

$$
\left|B_{p}\right\rangle=\frac{\Omega_{1, p}|b\rangle+\Omega_{2, p+Q}|c\rangle}{\sqrt{\Omega_{1, p}^{2}+\Omega_{2, p+Q}^{2}}} .
$$

If the pulses are short, then the states are the same,

$$
\left|B_{p}\right\rangle \simeq|B\rangle,
$$

and it is a bright state which is driven by a train of pulses with effective Rabi frequency

$$
\Omega_{\mathrm{eff}}=\sqrt{\Omega_{1, p}^{2}+\Omega_{2, p+Q}^{2}} .
$$

The states orthogonal to the bright one are the excited state $|a\rangle$ and the dark state given by

$$
|D\rangle=\frac{\Omega_{2, p+Q}|b\rangle-\Omega_{1, p}|c\rangle}{\sqrt{\Omega_{1, p}^{2}+\Omega_{2, p+Q}^{2}}} ;
$$

the latter is decoupled from the electromagnetic field. The system interacting with the field ends up in this state and does not absorb radiation.

\section{DISCUSSION}

We have shown that a comb of short pulses can induce atomic coherence in a resonance atomic medium, and this coherence leads to electromagnetically induced transparency. The width of the EIT resonance is determined by the relaxation rate of coherence between ground states $|b\rangle$ and $|c\rangle$, and it would be dramatically reduced if an appropriate $\mathrm{Rb}$ cell with a buffer gas were used. In the literature the observation of an EIT width below $40 \mathrm{~Hz}$ is reported in Ref. [5]. It confirms that mode-locked lasers can be used to create narrow EIT resonances, and in the next step the repetition rate should be locked to the EIT resonance frequency.

The narrow transparency resonances obtained here with a comb of short pulses can be used for most applications where EIT has advantages, for example, magnetometery $[6,10]$ atomic clocks $[8,9]$, and frequency chains $[7,26]$.

\section{SUMMARY}

In summary, a technique based on a mode-locked laser was applied to observe narrow EIT resonances in rubidium vapor. This technique can be used for realization of alloptical reference frequency chains by using femtosecond lasers. Another application might be the realization of a compact atomic clock with a mode-locked diode laser. In this case a fiber may be used as the external cavity.

\section{ACKNOWLEDGMENTS}

We would like to thank M. A. Gubin, J. Kitching, P. Hemmer, and V. L. Velichansky for useful and stimulating discussions. This work was supported by the Office of Naval Research, the Air Force Research Laboratory (Rome, NY), the National Science Foundation, the Defense Advanced Research Projects Agency QuIST, Texas A\&M University Telecommunication and Information Task Force (TITF) Initiative, and the Welch Foundation (Grant No. A1261).
[1] O. A. Kocharovskaya and Y. I. Khanin, Zh. Eksp. Teor. Fiz. 90, 1610 (1986) [Sov. Phys. JETP 63, 945 (1986)].

[2] E. Arimondo, in Progress in Optics, edited by E. Wolf (Elsevier, Amsterdam, 1996), Vol. 35, pp. 257-354; S. E. Harris, Phys. Today7, 36 (1997).

[3] K. J. Boller, A. Imamoglu, and S. E. Harris, Phys. Rev. Lett. 66, 1360 (1992); O. A. Kocharovskaya and Ya. I. Khanin, Pis'ma Zh. Eksp. Teor. Fiz. 48, 581 (1988) [JETP Lett. 48, 630 (1988)].

[4] M. D. Lukin, M. Fleischhauer, A. S. Zibrov, H. G. Robinson, V. L. Velichansky, L. Hollberg, and M. O. Scully, Phys. Rev. Lett. 79, 2959 (1997).

[5] S. Brandt, A. Nagel, R. Wynands, and D. Meschede, Phys. Rev. A 56, R1063 (1997).

[6] M. O. Scully, Phys. Rev. Lett. 67, 1855 (1991); M. O. Scully and M. Fleischhauer, ibid. 69, 1360 (1992).

[7] L. Arissian et al., J. Mod. Opt. 49, 2517 (2002).

[8] J. Kitching et al., Electron. Lett. 37, 1449 (2001); J. Kitching et al., J. Opt. Soc. Am. B 18, 1676 (2001).

[9] S. Knappe et al., Appl. Phys. Lett. 85, 1460 (2004).

[10] C. Affolderbach et al., Appl. Phys. B: Lasers Opt. 75, 605 (2002); M. Stahler et al. Europhys. Lett. 54, 323 (2001).

[11] S. E. Harris and L. V. Hau, Phys. Rev. Lett. 82, 4611 (1999).
[12] S. E. Harris and Y. Yamamoto, Phys. Rev. Lett. 81, 3611 (1998).

[13] A. Imamoğlu, H. Schmidt, G. Woods, and M. Deutsch, Phys. Rev. Lett. 79, 1467 (1997); M. Dunstan, S. Rebic, S. Tan, S. Parkins, M. Collett, and D. Walls, in Proceedings: Quantum Communication, Computing, and Measurement 2, edited by $\mathrm{P}$. Kumar, G. M. D’Ariano, and O. Hirota (unpublished); M. D. Lukin, A. Matsko, M. Fleischhauer, and M. O. Scully, Phys. Rev. Lett. 82, 1847 (1999).

[14] M. Jain, G. Y. Yin, J. E. Field, and S. E. Harris, Opt. Lett. 18, 998 (1993).

[15] H. Schmidt and A. Imamoğlu, Opt. Lett. 21, 1936 (1996).

[16] G. Alzetta, A. Gozzini, L. Moi, and G. Orriols, Nuovo Cimento Soc. Ital. Fis., B 36, 5 (1976).

[17] S. Brattke, U. Kallmann, and W. D. Hartmann, Eur. Phys. J. D 3, 159 (1998).

[18] K. J.. Boller, A. Imamoglu, and S. E. Harris, Phys. Rev. Lett. 66, 2593 (1993); 72, 52 (1994).

[19] A. S. Zibrov et al., Phys. Rev. Lett. 76, 3935 (1996).

[20] S. Harris and A. Sokolov, Phys. Rev. Lett. 81, 2894 (1998).

[21] J. Kitching and L. Hollberg, Phys. Rev. A 59, 4685 (1999).

[22] B. S. Ham, P. R. Hemmer, and M. S. Shahriar, Opt. Commun. 144, 227 (1997); B. S. Ham, M. S. Shahriar, and P. R. Hem- 
mer, Opt. Lett. 22, 1138 (1997).

[23] A. Imamoglu, Opt. Commun. 179, 179 (2000); D. E. Nikonov, A. Imamoglu, and M. O. Scully, Phys. Rev. B 59, 12212 (1999).

[24] C. J. Wei and N. B. Manson, Phys. Rev. A 60, 2540 (1999).

[25] R. Coussement, Yu. Rostovtsev, J. Odeurs, G. Neyens, P. Mandel, G. Kozyreff, R. Shakhmuratov, and O. Kocharovskaya,
Phys. Rev. Lett. 89, 107601 (2002).

[26] S. A. Diddams et al., Phys. Rev. Lett. 84, 5102 (2000); T. Utem, R. Holzwarth, and T. W. Hansch, Nature (London) 416, 233 (2002).

[27] P. P. Vasilev, Opt. Quantum Electron. 24, 801 (1992).

[28] M. G. Littman and H. J. Metcalf, Appl. Opt. 17, 2224 (1978);

K. C. Harvey and C. J. Myatt, Opt. Lett. 16, 910 (1991). 